\title{
Atomistic Full-quantum and Full-band Transport Model for Zigzag Group-IVA Nanoribbon-based Structures with Noniterative Calculation Framework
}

\author{
Win-Jet Luo, ${ }^{1}$ Wei-Ta Chien, ${ }^{2}$ Hau-Chen Yen, ${ }^{3}$ and Chun-Nan Chen ${ }^{4}$ \\ ${ }^{1}$ Department of Refrigeration, Air Conditioning and Energy Engineering, \\ National Chin-Yi University of Technology, Taichung 411, Taiwan \\ ${ }^{2}$ Department of Refrigeration \& Air Conditioning and Energy, Far East University, Tainan 74448, Taiwan \\ ${ }^{3}$ Department of Electrical Engineering, Far East University, Tainan 74448, Taiwan \\ ${ }^{4}$ Quantum Engineering Laboratory, Department of Physics, \\ Tamkang University, Tamsui, New Taipei 25137, Taiwan
}

(Received June 3, 2020; accepted October 21, 2020)

Keywords: complex energy band, group-IVA nanoribbon, quantum transport, nonequilibrium Green's function (NEGF), full band, full quantum, spin-orbit coupling, noniterative calculation

In this paper, we expand our previous paper [Mod. Phys. Lett. B 32 (2018) 1750355] from the calculation of planar graphene with one band $\left(p_{z}\right)$ to the calculation of two-dimensional buckled group-IVA materials with multiple bands $\left(S, p_{x}, p_{y}\right.$, and $\left.p_{z}\right)$; thus, the proposed method is a fullband model. Furthermore, the proposed method is established using a nonequilibrium Green's function (NEGF) method in association with the complex energy-band technique, so it is in the full-quantum framework. Unlike other methods, the proposed method is noniterative and thus computationally cost-efficient.

\section{Introduction}

The graphene, which is regarded as a semimetal or zero-gap semiconductor, possesses a twodimensional (2D) honeycomb structure of carbon atoms, and it has attracted tremendous interest owing to its unique properties. ${ }^{(1-3)}$ Therefore, graphene is considered as a promising material for future research. However, its zero energy gap has been widely regarded as an obstacle to its application in next-generation nanoelectronics. Therefore, material researchers have tried to solve the zero-gap problem by using new $2 \mathrm{D}$ honeycomb materials from other elements in group-IVA of the periodic table. Firstly, many investigations have shown that $2 \mathrm{D}$ silicene ${ }^{(4,5)}$ and germanene, ${ }^{(4,5)}$ which are the graphene-like analogues of silicon and germanium, respectively, have promising applications in nanoscale electronic devices. The last research in this field is related to the 2D honeycomb lattice of a Sn monolayer, commonly referred to as stanene. ${ }^{(4,6)}$

Graphene possesses a planar 2D honeycomb lattice of carbon atoms with $\pi$-bonding. In contrast to graphene, other 2D layered group-IVA materials possess a buckled structure in the honeycomb lattice, which leads to partial $s p^{3}$ hybridization of an electronic configuration. ${ }^{(4)}$ The buckling of a honeycomb lattice enhances the hybridization between $\pi$ and $\sigma$ orbitals. $^{(6)}$ *Corresponding author: e-mail: quantum@mail.tku.edu.tw https://doi.org/10.18494/SAM.2020.3105 
Notably, owing to the enhancement of $s p^{3}$ hybridization with increasing group-IVA atomic number, out-of-plane buckling of the honeycomb lattice is enhanced. Buckled 2D materials possess a stronger spin-orbit coupling (SOC) interaction, which gives rise to a band gap between conduction and valence bands. ${ }^{(6,7)}$ A small band gap is one of the most important differences of silicene and germanene from graphene. ${ }^{(8)}$ For stanene, its relatively large buckling and thus its stronger SOC interaction open an obvious energy gap in the energy band diagram, ${ }^{(7)}$ which solves the zero-gap problem for planar 2D nanostructures.

The nonequilibrium Green's function $(\mathrm{NEGF})^{(9-13)}$ method is the most powerful method for solving the quantum transport problems of nanoscale electronic devices. In the past, many studies have shown that the NEGF can be solved by a recursive (or iterative) technique. ${ }^{(14-18)} \mathrm{A}$ recursive technique may finish this job or not (divergence), while one of the disadvantages of this technique is the slow convergence in the iterative steps. Moreover, another technique for obtaining the NEGF is based on the calculation framework of the Dyson equation, which was proposed by Caroli et al. ${ }^{(19)}$ and others. ${ }^{(20-22)}$ One of the disadvantages of the Dyson equation is the difficulty to dispose the miscellaneous boundary conditions. However, the disadvantages of the recursive and Dyson techniques can be overcome by the method proposed in this paper, which a complex energy-band framework in association with the NEGF approach. ${ }^{(23-26)}$

In this paper, the properties of zigzag-edged group-IVA nanoribbon (z4ANR) structures such as the density of states (DOS), transmission coefficient, and conductance will be explored by the proposed technique.

\section{Theoretical Methods}

The z4ANR sample considered in this paper possesses $N$ zigzag lines and has a central channel region composed of $l$ atomic layers denoted by $\sigma=1,2, \ldots, l$, as shown in Fig. 1 . It is supposed that flat-band cases occur in the left-side incoming $(\sigma \leq 0)$ and right-side outgoing $(\sigma \geq l+1)$ regions, which are located outside of the central channel region.

In this study, the theoretical model 4 of a monolayer group-IVA element structure is a tightbinding calculation based on the $\alpha_{\beta}^{\tau}(\alpha=s, x, y, z)$ orbital, where $\beta$ is an $A$ or $B$ sublattice, and the electron spin $\tau$ is along the up ( $\uparrow)$ or down $(\downarrow)$ direction. Therefore, the Hamiltonian of these monolayer group-IVA elements can be expressed in $16 \times 16$ matrix form with the $\alpha_{\beta}^{\tau}$ orbital, which is presented in Appendix A.

\subsection{Hamiltonian matrix and state function of z4ANRs}

In the flat-band case, the state function $\left|k_{\perp}\right\rangle$ of a z4ANR, which has $N$ zigzag lines, is a combination of the $16 N$ tight-binding basis $\mid k_{\perp}, j, \alpha_{\beta}^{\tau}>$, which can be written as

$$
\left|k_{\perp}>=\sum_{\tau=\uparrow \downarrow} \sum_{\alpha=s, x, y, z} \sum_{j=1}^{N} \sum_{\beta=A, B} b_{j, \alpha, \beta, \tau}\left(k_{\perp}\right)\right| k_{\perp}, j, \alpha_{\beta}^{\tau}>,
$$

where $b_{j, \alpha, \beta, \tau}$ specifies the expansion coefficient, $\alpha_{\beta}^{\tau}$ denotes the $\alpha$ orbital of the group-IVA element with electron spin $\tau(=\uparrow$ or $\downarrow)$ located at sublattice $\beta(=A$ or $B), j$ is the index of the $N$ 


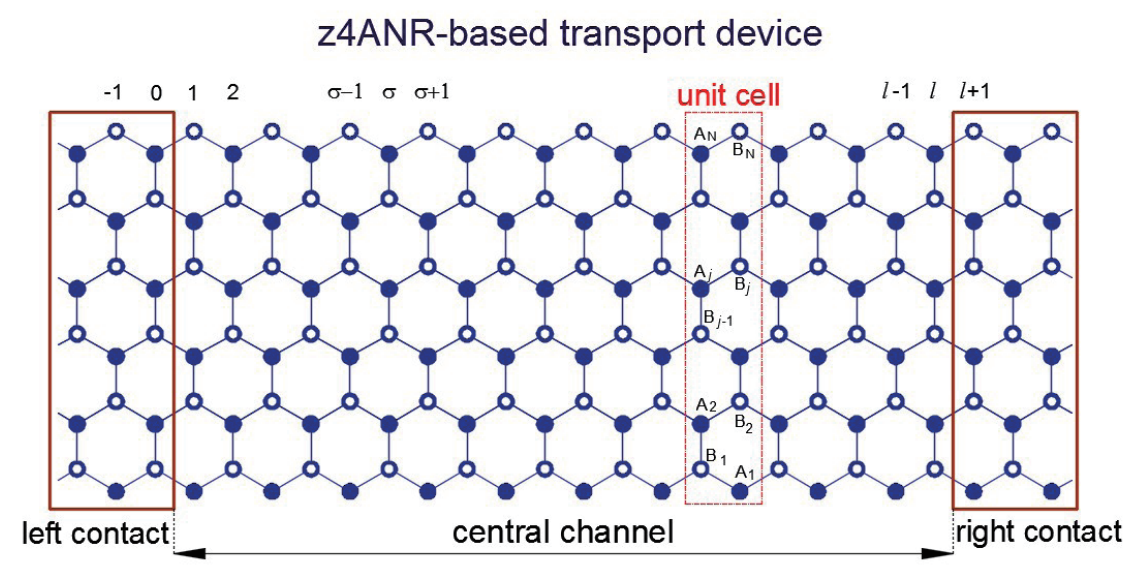

Fig. 1. (Color online) Geometric structure of z4ANR-based transport device (width, $N$ zigzag lines; channel length, $l$ atomic layers).

adjacent zigzag lines in the transverse direction $(\|)$ of the z4ANR, and $k_{\perp}$ is the wave vector directed along the z4ANR direction $(\perp)$. The electron energy $E$, which is shown as $\mid k_{\perp}, E>$ and $b_{j, \alpha, \beta, \tau}\left(k_{\perp}, E\right)$, is omitted for brevity here. Furthermore, the tight-binding basis of a z4ANR Hamiltonian can be expressed as

$$
\left|k_{\perp}, j, \alpha_{\beta}^{\tau}>=\frac{1}{\sqrt{\Omega}} \sum_{\sigma} \exp \left(i k_{\perp} \sigma a^{\prime}\right)\right| \sigma, j, \alpha_{\beta}^{\tau}>
$$

where $\Omega$ denotes the normalization number, $\sigma$ specifies a layer label increasing along the $\perp$ direction, $a^{\prime}$ denotes the spacing of two neighboring layers, and $\mid \sigma, j, \alpha_{\beta}^{\tau}>$ denotes the $\alpha_{\beta}^{\tau}$ orbital of the group-IVA element at lattice site $(\sigma, j)$. In the expression of the $\mid k_{\perp}, j, \alpha_{\beta}^{\tau}>$ basis, the z4ANR Hamiltonian $H_{z 4 A N R}\left(k_{\perp}\right)$, which possesses $16 N \times 16 N$ matrix formalism, is written in Appendix B. The state function of a z4ANR can also be expressed in the form of

$$
\left|k_{\perp}>=\sum_{\sigma} \sum_{\alpha, \tau} \sum_{j, \beta} c_{\sigma, j, \alpha, \beta, \tau}\left(k_{\perp}\right)\right| \sigma, j, \alpha_{\beta}^{\tau}>
$$

where $c_{\sigma, j, \alpha, \beta, \tau}\left(k_{\perp}\right)=\frac{1}{\sqrt{\Omega}} b_{j, \alpha, \beta, \tau}\left(k_{\perp}\right) \exp \left(i k_{\perp} \sigma a^{\prime}\right)$. On the basis of the structure of z4ANR lattice points, the Hamiltonian of a z4ANR in the nearest-neighbor approach can also be expressed as

$$
H_{z 4 A N R}\left(k_{\perp}\right)=H_{\sigma, \sigma-1} e^{-i k_{\perp} a^{\prime}}+H_{\sigma, \sigma}+H_{\sigma, \sigma+1} e^{+i k_{\perp} a^{\prime}},
$$

where $H_{\sigma, \sigma}$ and $H_{\sigma, \sigma \pm 1}$ are $16 N \times 16 N$ matrices (see Appendix C), whose elements are written as

$$
\left(H_{\sigma, \sigma}\right)_{j, \alpha, \beta, \tau ; j^{\prime}, \alpha^{\prime}, \beta^{\prime}, \tau^{\prime}}=<\sigma, j, \alpha_{\beta}^{\tau}|(H-E)| \sigma, j^{\prime}, \alpha_{\beta^{\prime}}^{\tau^{\prime}}>
$$


and

$$
\left(H_{\sigma, \sigma \pm 1}\right)_{j, \alpha, \beta, \tau ; j^{\prime}, \alpha^{\prime}, \beta^{\prime}, \tau^{\prime}}=<\sigma, j, \alpha_{\beta}^{\tau}|H| \sigma \pm 1, j^{\prime}, \alpha_{\beta^{\prime}}^{\tau^{\prime}}>
$$

respectively.

\subsection{Complex energy-band structure of z4ANRs}

The Schrödinger equation $(H-E) \mid k_{\perp}>=0$ in a z4ANR structure can be expressed as ${ }^{(27-29)}$

$$
\left[\begin{array}{cc}
0 & I \\
-H_{\sigma, \sigma+1}^{-1} H_{\sigma, \sigma-1} & -H_{\sigma, \sigma+1}^{-1} H_{\sigma, \sigma}
\end{array}\right]\left[\begin{array}{c}
c_{\sigma-1} \\
c_{\sigma}
\end{array}\right]=e^{i k_{\perp} a^{\prime}}\left[\begin{array}{c}
c_{\sigma-1} \\
c_{\sigma}
\end{array}\right],
$$

where $I$ denotes a $16 N \times 16 N$ identity matrix, a state function $\left|k_{\perp}\right\rangle$ specifies the available planewave states in the left and right regions, and $c_{\sigma}$ can be expressed as a $16 \mathrm{~N}$-length column vector of coefficients whose components are denoted as $c_{\sigma, j, \alpha, \beta, \tau}$, i.e.,

$$
c_{\sigma}=\left[\begin{array}{c}
c_{\sigma, 1} \\
c_{\sigma, 2} \\
\vdots \\
c_{\sigma, 16 N}
\end{array}\right] .
$$

According to Bloch's theorem, the tight-binding coefficients must satisfy the relation $c_{\sigma}=e^{i k_{\perp} a^{\prime}} c_{\sigma-1}$.

To solve the eigenvalue $\left(e^{i k_{\perp} a^{\prime}}\right)$ of Eq. (6), we can obtain a set of $2 \times(16 N)$ real or complex wave vectors $\left\{k_{\perp, \lambda} ; \lambda=1,2, \cdots, 32 N\right\}$ and their corresponding state functions $\left|k_{\perp, \lambda}\right\rangle$ for a given electron energy $E$, which can yield an $E-k_{\perp, \lambda=1-32 N}$ complex energy-band diagram. ${ }^{(23-25)}$

\subsection{Hamiltonian matrix and wave function of z4ANR-based devices}

In the z4ANR-based structure, the wave function of the Schrödinger equation $(H-E) \mid \psi>=0$ can be written as

$$
\left|\psi>=\sum_{k_{\perp}} a\left(k_{\perp}\right)\right| k_{\perp}>=\sum_{\sigma} \sum_{\alpha, \tau} \sum_{j, \beta} \sum_{k_{\perp}} a\left(k_{\perp}\right) c_{\sigma, j, \alpha, \beta, \tau}\left(k_{\perp}\right) \mid \sigma, j, \alpha_{\beta}^{\tau}>,
$$

where $a\left(k_{\perp}\right)$ denotes the amplitude coefficient of a z4ANR state function $\left|k_{\perp}\right\rangle$. Therefore, the wave function of the whole structure can be expressed in the form

$$
\left|\psi>=\sum_{\sigma} \sum_{\alpha, \tau} \sum_{j, \beta} f_{\sigma, j, \alpha, \beta, \tau}\right| \sigma, j, \alpha_{\beta}^{\tau}>,
$$

where $f_{\sigma, j, \alpha, \beta, \tau}$ is $\sum_{k_{\perp}} a\left(k_{\perp}\right) c_{\sigma, j, \alpha, \beta, \tau}\left(k_{\perp}\right)$, which is electron-energy-dependent, i.e., $f_{\sigma, j, \alpha, \beta, \tau}(E)$. 
The Schrödinger equation $(H-E) \mid \psi>=0$ can be expressed in the $\mid \sigma, j, \alpha_{\beta}^{\tau}>$ basis form, and thus we obtain the following combinative equation for the $\sigma$ th layer: ${ }^{(28-30)}$

$$
H_{\sigma, \sigma-1} f_{\sigma-1}+H_{\sigma, \sigma} f_{\sigma}+H_{\sigma, \sigma+1} f_{\sigma+1}=0
$$

where $H_{\sigma, \sigma}$ and $H_{\sigma, \sigma \pm 1}$ can be written in the form of $16 \mathrm{~N} \times 16 \mathrm{~N}$ matrices, as shown in Appendix $\mathrm{C}$, and $f_{\sigma}$ is a $16 N$-length column vector whose components are $f_{\sigma, j, \alpha, \beta, \tau}$. Therefore, the Hamiltonian matrix of the whole structure can be expressed as

$$
H-E \hat{I}=\left[\begin{array}{ccccccc}
\ddots & \ddots & \ddots & 0 & 0 & \vdots & 0 \\
0 & H_{\sigma-1, \sigma-2} & H_{\sigma-1, \sigma-1} & H_{\sigma-1, \sigma} & 0 & \vdots & \vdots \\
\vdots & 0 & H_{\sigma, \sigma-1} & H_{\sigma, \sigma} & H_{\sigma, \sigma+1} & 0 & \vdots \\
\vdots & \vdots & 0 & H_{\sigma+1, \sigma} & H_{\sigma+1, \sigma+1} & H_{\sigma+1, \sigma+2} & 0 \\
0 & \vdots & 0 & 0 & \ddots & \ddots & \ddots
\end{array}\right] .(11)
$$

\subsection{Boundary conditions solved by complex energy-band method}

We rearrange the state functions $\left|k_{\perp, \lambda=1 \sim 32 N}\right\rangle$ of the complex energy-band formalism, which are obtained from Eq. (6). Therefore, the index of $\lambda=1,2, \ldots, 16 \mathrm{~N}$ corresponds to the state functions, which either propagate ( $k_{\perp}$ real) or decay ( complex) to the right-hand side. On the other hand, $\lambda=16 N+1,16 N+2, \ldots, 32 N$ correspond to those which either propagate or decay to the left-hand side. ${ }^{(29,30)}$ The boundary conditions are such that we have a known incoming plane-wave state from the left contact, no incoming plane-wave state from the right contact, and unknown outgoing transmitted and reflected plane-wave states in the right and left contacts, respectively. For a given energy $E$ and for a given amplitude (here unity) of an incoming planewave-like state (denoted by $i$ ) from the left, the wave functions in the left $(L)$ and right $(R)$ contacts must satisfy the boundary conditions of this example, which can be expressed in terms of the state functions of the complex energy-band structure as follows: ${ }^{(29,30)}$

$$
|\psi ; L>=| \psi^{i}>+\left|\psi^{\Re}>=I_{i}\right| k_{\perp, i} ; L>+\sum_{\lambda=1}^{16 N} a\left(k_{\perp, \lambda+16 N} ; L\right) \mid k_{\perp, \lambda+16 N} ; L>
$$

and

$$
|\psi ; R>=| \psi^{\Im}>=\sum_{\lambda=1}^{16 N} a\left(k_{\perp, \lambda} ; R\right) \mid k_{\perp, \lambda} ; R>,
$$

where $I_{i}$ denotes the known amplitude (here unity) coefficient of the incoming plane-wavelike state function from the left contact, and $a\left(k_{\perp, \lambda} ; R\right)$ and $a\left(k_{\perp, \lambda+16 N} ; L\right)$ are the unknown amplitude coefficients of the transmitted and reflected state functions, respectively. For convenience, we use $\mathfrak{R}$ and $\mathfrak{I}$ to denote the outgoing waves that propagate (or decay) to the left in the left contact and to the right in the right contact, respectively. 

We rewrite Eq. (9) as $\left|\psi^{\zeta}>=\sum_{\sigma} \sum_{\alpha, \tau} \sum_{j, \beta} f_{\sigma, j, \alpha, \beta, \tau}^{\zeta}\right| \sigma, j, \alpha_{\beta}^{\tau}>$, then we obtain the
relation $^{(10,30,31)}$

$$
\left[f_{o}^{\zeta}\right]=\left[B^{\zeta}\right]\left[a^{\zeta}\right]
$$

where $\zeta$ denotes $\mathfrak{I}$ and $\mathfrak{R}$, which represent transmitted and reflected waves, respectively,

$$
\begin{aligned}
& {\left[B_{\sigma}^{\mathfrak{J}}\right]=\left[\begin{array}{llll}
c_{\sigma}\left(k_{\perp, 1}\right) & c_{\sigma}\left(k_{\perp, 2}\right) & \cdots & c_{\sigma}\left(k_{\perp, 16 N}\right)
\end{array}\right]_{R},} \\
& {\left[B_{\sigma}^{\Re}\right]=\left[\begin{array}{llll}
c_{\sigma}\left(k_{\perp, 16 N+1}\right) & c_{\sigma}\left(k_{\perp, 16 N+2}\right) & \cdots & \left.c_{\sigma}\left(k_{\perp, 32 N}\right)\right]_{L},
\end{array}\right.} \\
& a^{\mathfrak{I}}=\left[\begin{array}{c}
a\left(k_{\perp, 1}\right) \\
a\left(k_{\perp, 2}\right) \\
\vdots \\
a\left(k_{\perp, 16 N}\right)
\end{array}\right]_{R}, \\
& a^{\Re}=\left[\begin{array}{c}
a\left(k_{\perp, 16 N+1}\right) \\
a\left(k_{\perp, 16 N+2}\right) \\
\vdots \\
a\left(k_{\perp, 32 N}\right)
\end{array}\right]_{L} .
\end{aligned}
$$

The coefficients $f_{\sigma, j, \alpha, \beta, \tau}$ of the $\mid \sigma, j, \alpha_{\beta}^{\tau}>$ orbital in the right and left contacts can be acquired through Eqs. (13)-(15), which result in two boundary conditions, ${ }^{(10,30,31)}$

$$
\begin{gathered}
{\left[f_{l+2}^{\mathfrak{I}}\right]=\left[B_{l+2}^{\mathfrak{I}}\right]\left[B_{l+1}^{\mathfrak{I}}\right]^{-1}\left[f_{l+1}^{\mathfrak{I}}\right],} \\
{\left[f_{-1}^{\Re}\right]=\left[B_{-1}^{\Re}\right]\left[B_{0}^{\Re}\right]^{-1}\left[f_{0}^{\Re}\right] .}
\end{gathered}
$$

\subsection{Schrödinger-like equation in the NEGF form solved by complex energy-band method}

From Eqs. (11), (12), and (16), we can obtain the Schrödinger-like equation for the active region $(0 \leq \sigma \leq l+1)$ in the NEGF framework as ${ }^{(9,10,30,31)}$

$$
\left[H_{a c t}-E \hat{I}+\Sigma_{L}+\Sigma_{R}\right]\{\phi\}=\{S\},
$$

where the Hamiltonian in the active region $\left(H_{a c t}\right)$, the boundary self-energies for the left $(L)$ and right $(R)$ contacts $\left(\Sigma_{L, R}\right)$, the wave function $\{\phi\}$, and the source term $\{S\}$ are written in the matrix form as 


$$
\begin{gathered}
H_{a c t}-E \hat{I}=\left[\begin{array}{ccccc}
H_{0,0} & H_{0,1} & 0 & \vdots & 0 \\
H_{1,0} & H_{1,1} & H_{1,2} & 0 & \vdots \\
0 & \ddots & \ddots & \ddots & 0 \\
\vdots & 0 & H_{l, l-1} & H_{l, l} & H_{l, l+1} \\
0 & \vdots & 0 & H_{l+1, l} & H_{l+1, l+1}
\end{array}\right], \\
\Sigma_{L}=\left[H_{0,-1}\right]\left[B_{-1}^{\Re}\right]\left[B_{0}^{\Re}\right]^{-1}, \\
\Sigma_{R}=\left[H_{l+1, l+2}\right]\left[\begin{array}{c}
B_{l+2}^{\mathfrak{I}}
\end{array}\right]\left[B_{l+1}^{\mathfrak{I}}\right]^{-1}, \\
\{\phi\}=\left[\begin{array}{c}
\Re \\
f_{0}^{\Re} \\
f_{1} \\
\vdots \\
f_{l} \\
f_{l+1}^{\mathfrak{I}}
\end{array}\right]
\end{gathered}
$$

and

$$
\{S\}=\left[\begin{array}{c}
-\sum_{\sigma=-1}^{0} H_{0, \sigma} c_{\sigma, i} \\
-H_{1,0} c_{0, i} \\
0 \\
\vdots \\
0
\end{array}\right] .
$$

\subsection{Applicative calculation using NEGF framework}

The Green's function of the whole device is expressed as

$$
G_{d}=\left(E \hat{I}-H_{a c t}-\Sigma_{L}-\Sigma_{R}\right)^{-1} .
$$


The Green's function $G_{d}$ is obtained, and then the transmission function $\bar{T}(E)$ is determined by the trace of ${ }^{(12,32)}$

$$
\bar{T}(E)=T(E) M(E)=\operatorname{Tr}\left[\Gamma_{L} G_{d} \Gamma_{R} G_{d}^{+}\right],
$$

where $\bar{T}(E)$ is the product of the number of forward-propagating eigenstates $M(E)$ and the transmission probability $T(E), \Gamma_{L, R}=i\left(\Sigma_{L, R}-\Sigma_{L, R}^{+}\right)$is the broadening factor, superscript ' + ' is the complex conjugate transpose, and $\operatorname{Tr}$ denotes the trace operator. Occasionally, $M(E)$ is referred to as the propagating channel or propagating mode.

The conductance $G(E)$ of the device is obtained from the Fermi energy $E_{F}$, and it can be associated with the transmission function $\bar{T}(E)$ as ${ }^{(12,32-34)}$

$$
G\left(E_{F}\right)=\frac{2 e^{2}}{h} \bar{T}\left(E_{F}\right)
$$

where $h$ denotes the Planck constant, $e$ denotes the electron charge, and $2 e^{2} / h$ represents the conductance quantum. Furthermore, the transmission function $\bar{T}(E)$ should be obtained at the Fermi energy $E_{F}$ using $\bar{T}\left(E_{F}\right)=\left.\operatorname{Tr}\left[\Gamma_{L} G_{d} \Gamma_{R} G_{d}^{+}\right]\right|_{E=E_{F}}$.

If the Green's function $G_{d}$ is obtained, the DOS can be determined via ${ }^{(12,15,32)}$

$$
\operatorname{DOS}(E)=\frac{1}{2 \pi} \operatorname{Tr}\left[G_{d}\left(\Gamma_{L}+\Gamma_{R}\right) G_{d}^{+}\right]
$$

\section{Results and Discussion}

Figures 2(a)-2(d) show the energy-band structures of monolayer group-IVA elements, which are calculated by the tight-binding four-band technique with the SOC effect in the nearestneighbor approach. Furthermore, the tight-binding four-band results are in good agreement with the first-principles results only for the valence and conduction bands in the low-energy sections, ${ }^{(4)}$ i.e., near the $K$ points. Therefore, the four-band model used here can capture the essential physics of monolayer group-IVA elements in the low-energy region reasonably well.

Graphene possesses a planar $\left(\theta=90^{\circ}\right)$ honeycomb lattice of carbon atoms, where $\theta$ is the angle between the bond and the $z$ direction. In contrast to graphene, other layered group-IVA materials possess a buckled $\left(\theta \neq 90^{\circ}\right)$ structure in the honeycomb lattice. $\theta$ is $101.7^{\circ}$ for silicene, $106.5^{\circ}$ for germanene, and $107.1^{\circ}$ for stanene. ${ }^{(4)}$ The buckling in the honeycomb lattice enhances the overlap between $\pi$ and $\sigma$ orbitals. Therefore, the buckling in the honeycomb lattice leads to partial hybridization between $\pi$ and $\sigma$ orbitals, and thus partial $s p^{3}$ hybridization exists in the orbital configuration. Furthermore, owing to the increasing $s p^{3}$ hybridization with increasing group-IVA atomic number, the out-of-plane buckling of the honeycomb lattice is enhanced. Owing to the low atomic mass of graphene, its intrinsic SOC interaction is negligible. As shown 
in Appendix A, the strength of the SOC interaction $\xi_{0}$ is 0.009 for graphene, 0.034 for silicene, 0.196 for germanene, and 0.8 for stanene in units of $\mathrm{eV}^{(4)}$ Compared with the planar graphene, the buckled 2D group-IVA elements possess a stronger intrinsic SOC interaction, which gives rise to a band gap in the $K$ points. Possessing a small band gap is the most important difference of silicene and germanene from graphene, as shown in Figs. 2(a)-2(c). Compared with silicene and germanene, stanene possesses a larger buckled altitude and thus a stronger SOC interaction, which opens an obvious band gap in the energy-band diagram, as shown in Fig. 2(d).

A z4ANR, which has $N$ zigzag lines (width), possesses $2 N$ group-IVA atoms in the interior of its unit cell, as presented in Fig. 1. A $2 N$-center tight-binding framework of this case yields a $16 N \times 16 N$ [where $16 N=2 N \times 4(\because \alpha) \times 2(\because \tau)]$-dimensional Hamiltonian matrix, as presented in Appendix B. In the case of $N(=4)$ zigzag lines, the energy-band structures of the z4ANR members (zGNR, zSiNR, zGeNR, and zSnNR, which are constituted of graphene, silicene, germanene, and stanene, respectively) are computed and presented in Figs. 3(a)-3(d), respectively. The energy-band structure of a zGNR with $N(=4)$ zigzag lines is computed and

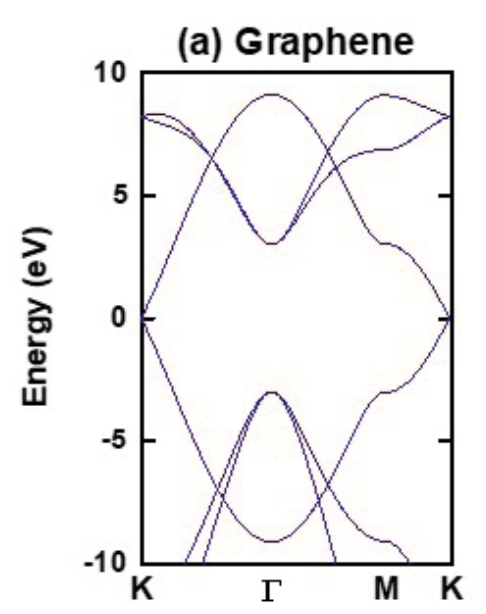

(b) Silicene

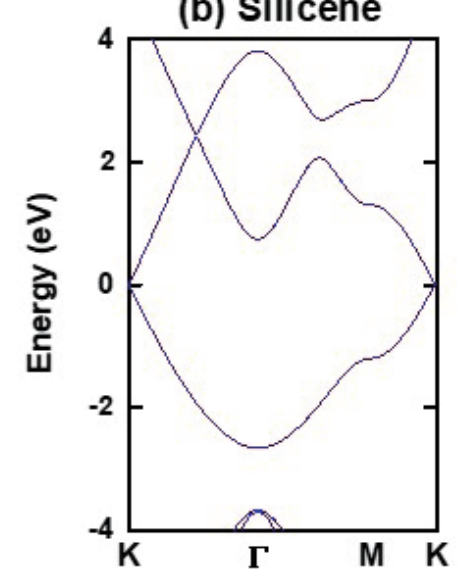

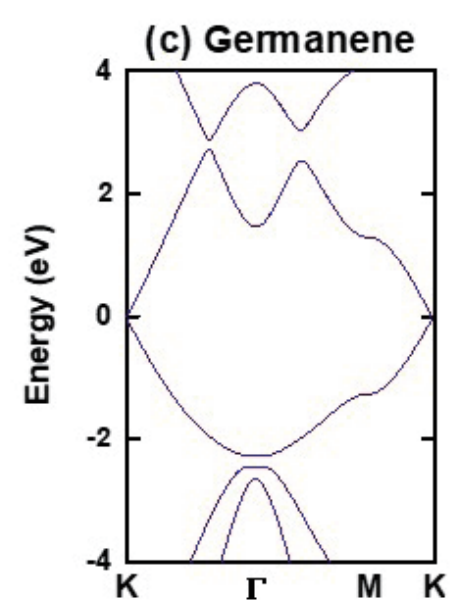

(d) Stanene

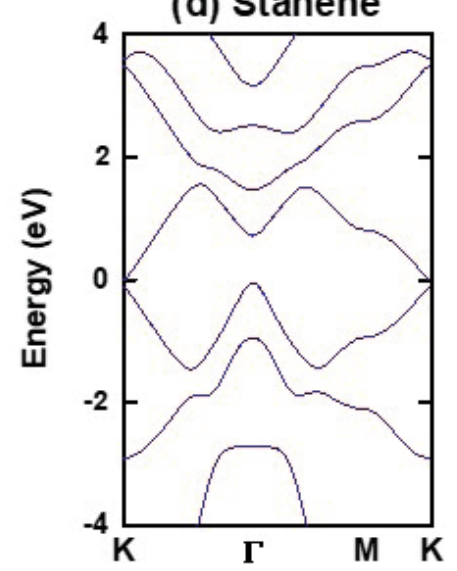

Fig. 2. (Color online) Energy-band diagrams for monolayer (a) graphene, (b) silicene, (c) germanene, and (d) stanene. 
(a) ZGNR

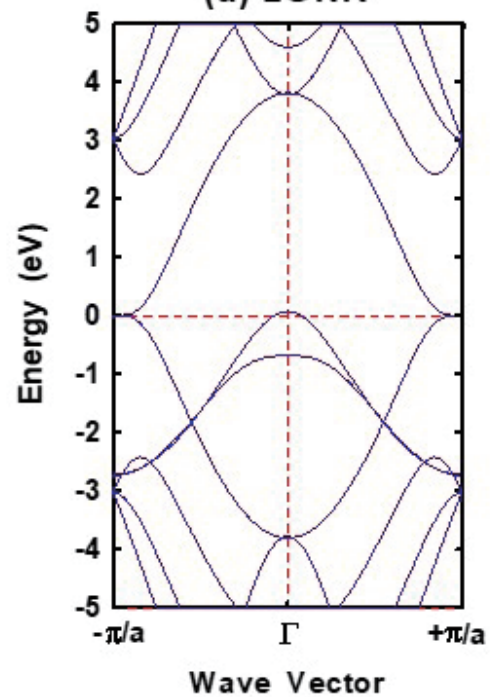

(b) zSiNR

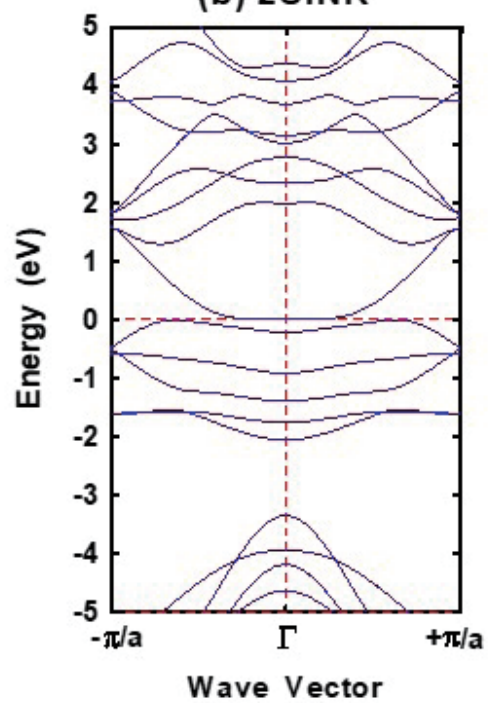

(c) zGeNR

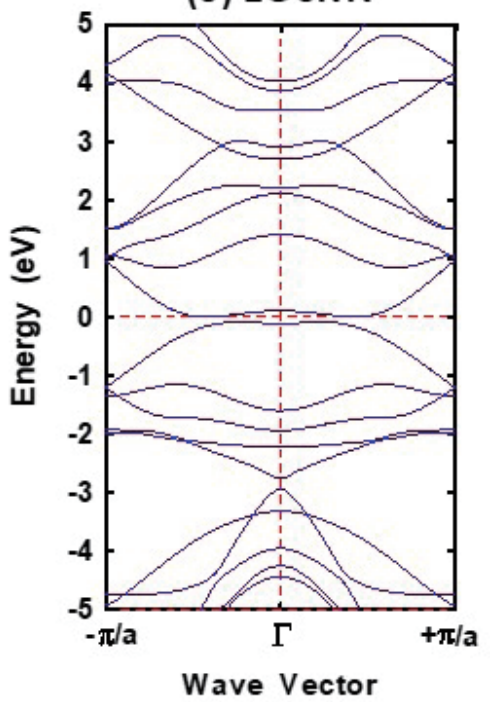

(d) $\mathrm{zSnNR}$

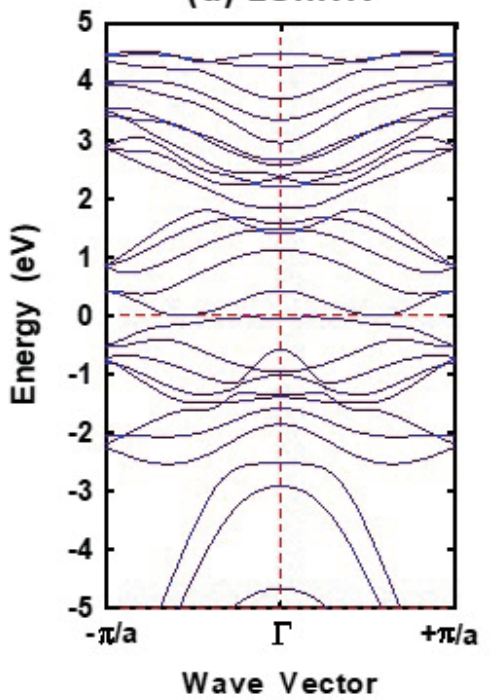

Fig. 3. (Color online) Energy-band diagram for single-layer (a) zGNR, (b) zSiNR, (c) zGeNR, and (d) zSnNR with $N(=4)$ zigzag lines.

presented in the left panel of Fig. 4, and its corresponding number of forward-propagating channels $M(E)$ is presented in the right panel.

Equation (6), which is an eigenvalue equation, possesses a $32 N \times 32 N$ matrix formalism. According to Eq. (6), at a certain energy $E$, one can attain $32 N$ real or complex eigenvalues $\left\{k_{\perp, \lambda} ; \lambda=1,2, \cdots, 32 N\right\}$ and their corresponding eigenvectors $\left|k_{\perp, \lambda}\right\rangle$, which can yield an $E-k_{\perp, \lambda=1 \sim 32 N}$ complex energy-band diagram. ${ }^{(23-25)}$ Moreover, some value of $E$ and its corresponding real $k_{\perp}$ yield the traditional $E-k_{\perp}$ energy-band diagram of a z4ANR. For example, the traditional $E-k_{\perp}$ energy-band diagram of a zGNR with $N(=4)$ zigzag lines is presented in the left panel of Fig. 4 . For a real value of $k_{\perp}$, the propagating waves transmit to 

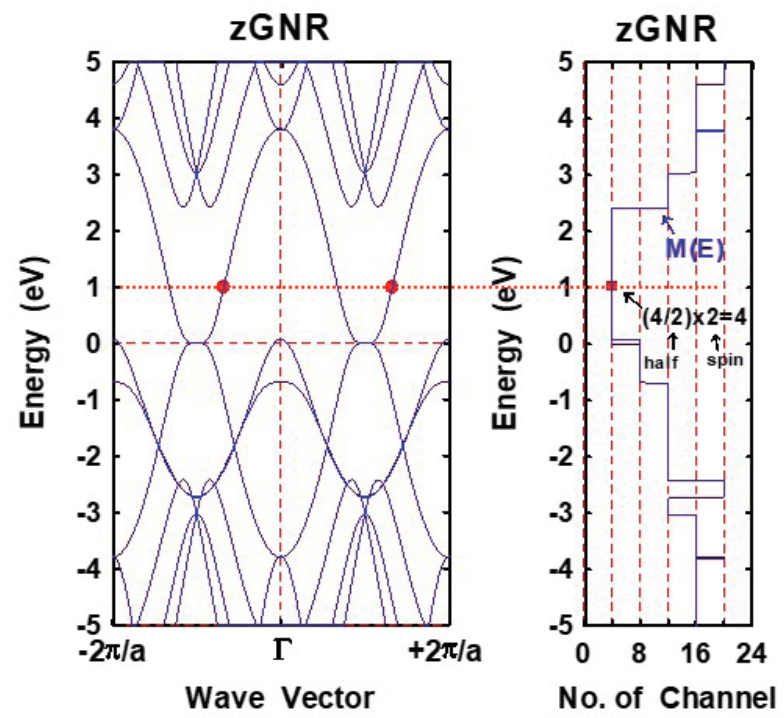

Fig. 4. (Color online) Energy-band diagram for single-layer zGNR with $N(=4)$ zigzag lines and its corresponding number of propagating channels at given energy $E$.

the right $\left[v_{g}\left(k_{\perp}\right)>0\right]$ or left $\left[v_{g}\left(k_{\perp}\right)<0\right]$ direction (see Fig. 4), where $v_{g}$ is the group velocity $\left[\hbar^{-1} \partial H_{N R B}\left(k_{\perp}\right) / \partial k_{\perp}\right]$. Furthermore, for a complex value of $k_{\perp}$, the evanescent waves decay exponentially in the right $\left[\operatorname{Im}\left(k_{\perp}\right)>0\right]$ or left $\left(\operatorname{Im}\left(k_{\perp}\right)<0\right)$ direction. Each of the eigenvectors of Eq. (6) corresponds to a pair of $k_{\perp}$ and $-k_{\perp}$; hence, half (16N) of the propagating or evanescent waves propagate rightward and the other half $(16 N)$ propagate leftward at a certain energy $E$, as shown in Fig. 4.

In the z4ANR-based devices considered in this section, the width of the channel is $N(=4)$ zigzag lines and the length of central channel is $l(=14)$ atomic layers. For these z4ANR-based samples, which assume a perfect flat-band potential profile, Figs. 5(a)-5(d) display the spectral diagrams of the transmission function $(\bar{T})$, conductance $(G)$, and $D O S$ as a function of energy $E$. Furthermore, for these z4ANR-based samples, which now have a double-barrier structure (DBS) potential profile imposed on them (i.e., central channel length: $l=2$ (barrier) +10 (well) +2 (barrier) $=14$ atomic layers with barrier height $0.7 \mathrm{eV}$ ), Figs. 6(a)-6(d) display the spectral diagrams of $\bar{T}, G$, and $D O S$ as a function of $E$.

Assuming a perfect flat-band potential, we have $T(E)=1$, and thus $\bar{T}(E)=M(E)$, for the z4ANR-based samples. According to calculations using Eqs. (23) and (24), these flat-band samples have a staircase-like conductance, which is the number of propagating channels $M(E)$ multiplied by the conductance quantum $2 e^{2} / h$, as displayed in the right panel of Fig. 4 and Figs. 5(a)-5(d). ${ }^{(35-37)}$ As presented in Figs. 5(a)-5(d) and Figs. 6(a)-6(d), it can be seen that in the DBS imposition, the quantization staircases of conductance spectra are destroyed and show more complicated oscillation behaviors due to the scattering from the DBSs. Namely, it is shown that the transition of conductance is from the quantized conductance in flat-band structures to resonant-tunneling conductance in DBSs. 
(a) ZGNR

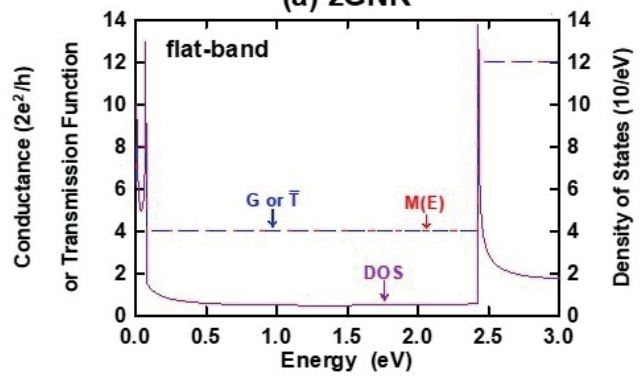

(c) zGeNR

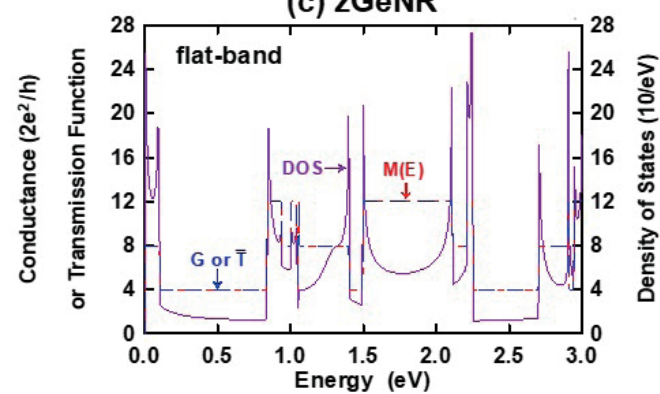

(b) zSiNR

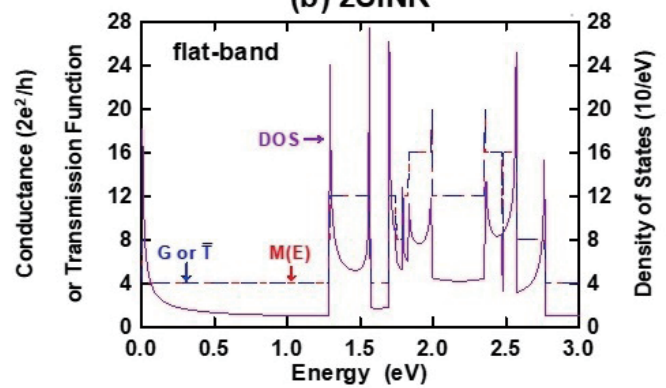

(d) zSnNR

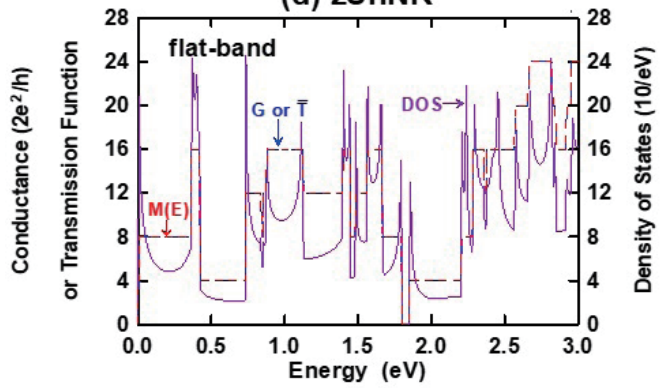

Fig. 5. (Color online) Transmission function $(\bar{T})$, conductance $(G)$, and density of states $(D O S)$ as functions of electron energy $E$ for the flat-band (a) zGNR-based, (b) zSiNR-based, (c) zGeNR-based, and (d) zSnNR-based device structures (width : $N=4$ zigzag lines; channel length $: l=14$ atomic layers), where $M(E)$ denotes the number of propagating channels at a given energy $E$.

\section{(a) ZGNR}

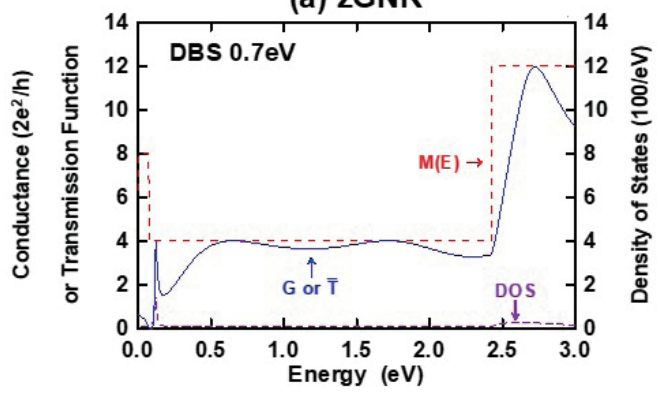

(c) zGeNR

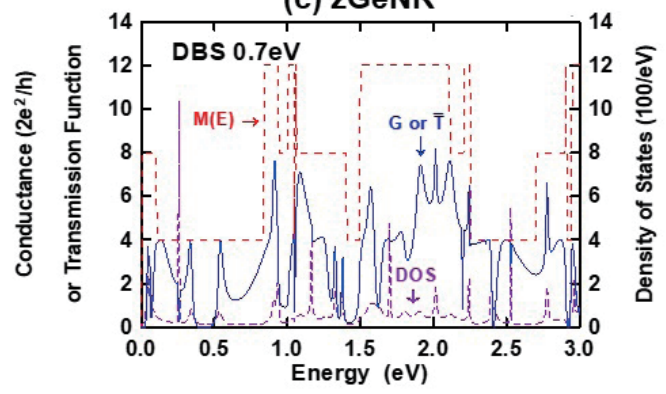

(b) ZSiNR

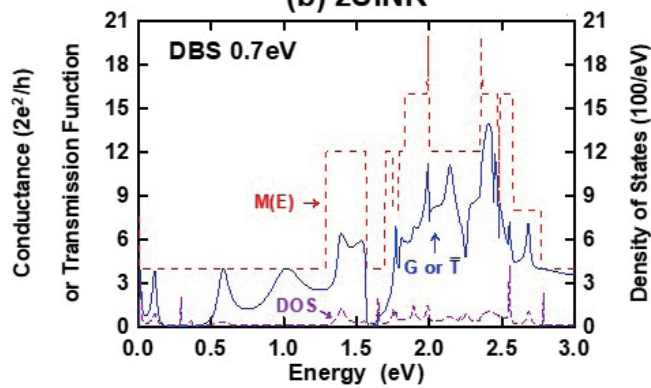

(d) ZSnNR

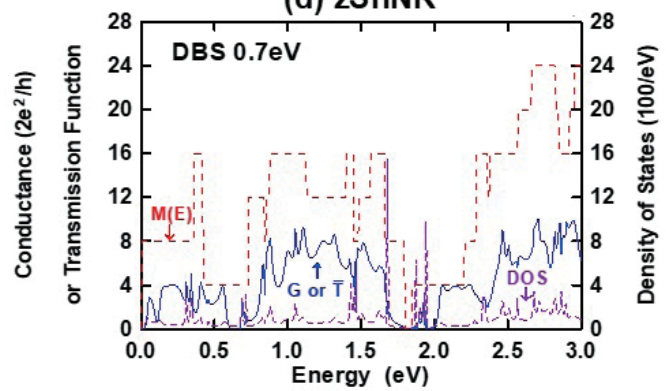

Fig. 6. (Color online) Transmission function $(\bar{T})$, conductance $(G)$, and density of states $(D O S)$ as functions of electron energy $E$ for z4ANR-based device structure (width : $N=4$ zigzag lines; channel length $: l=2+10+2=14$ atomic layers). A 2-10-2 atomic-layer DBS potential profile with barrier height $0.7 \mathrm{eV}$ is imposed on the (a) zGNRbased, (b) zSiNR-based, (c) zGeNR-based, and (d) zSnNR-based structures, where $M(E)$ denotes the number of propagating channels at a given energy $E$. 
A van Hove singularity can be seen as a singularity (nonsmooth point) in the DOS spectra of crystalline solids. The van Hove singularities occur where the derivative of the DOS with respect to energy $E$ diverges, which yields a strikingly sharp peak in the DOS spectra. ${ }^{(38-43)}$ Therefore, in a perfect flat-band z4ANR, the DOS diverges at the onset of each subband, as shown in Figs. 5(a)-5(d) and Figs. 3(a)-3(d). Figures 5(a)-5(d) display the strikingly sharp and asymmetric peaks in the DOS spectra, which are also called van Hove singularities.

In quantum transport devices such as DBSs, a series of discrete and fine confined levels (or states) exist in the central channel, and a continuous distribution of states exists in the left and right contacts. When the central channel and the two contacts are joined, the discrete channel levels couple and then acquire a few states of the two contacts. ${ }^{(12,32)}$ Therefore, the effect of coupling is to broaden the DOS in the central channel from its original discrete lines to a continuous spectrum, ${ }^{(12,32)}$ as shown in Figs. 6(a)-6(d). With increasing DBS barrier height, the coupling strength of the two contacts to the central channel decreases, and thus the line-shape sharpness and depth of the DOS peaks and dips increase. Therefore, the DBS has been verified to be a suitable structure for exploring the coupling strength of contacts to a central channel, and an increase in DBS barrier height yields the DOS modification of the central channel. As shown in Figs. 6(a)-6(d), when energy $E$ coincides with any resonant-tunneling confined levels of the DBS, irregularly spaced resonant-tunneling peaks occur in DOS spectra.

\section{Conclusions}

In this paper, we have developed an atomistic full-band and full-quantum model for calculating the electronic transport characteristics of z4ANR-based devices, which also involves the SOC effect. We have derived in detail the theoretical expressions for z4ANR-based devices such as their wave function, Hamiltonian matrix, and the Schrödinger-like equation in the NEGF form, which is based on the complex energy-band method. The proposed method is straightforward, nonrecursive, and thus computationally cost-efficient. Using the developed method, we have calculated and obtained important findings on z4ANR-based devices, such as their conductance quantization, van Hove singularities in the DOS, and the effect of contact interactions on the channel.

\section{Acknowledgments}

This work was supported in part by National Science Council (NSC), Taiwan, ROC, under Contract NSC-108-2112-M-032-015.

\section{References}

1 L. E. F. F. Torres, S. Roche and J. C. Charlier: Introduction to Graphene-Based Nanomaterials: From Electronic Structure to Quantum Transport (Cambridge University Press, Cambridge, U.K., 2014).

2 A. H. C. Neto, F. Guinea, N. M. R. Peres, K. S. Novoselov, and A. K. Geim: Rev. Mod. Phys. 81 (2009) 109. https://doi.org/10.1103/RevModPhys.81.109

3 K. S. Novoselov, A. K. Geim, S. V. Morozov, D. Jiang, Y. Zhang, S. V. Dubonos, I. V. Grigorieva, and A. A. Firsov: Science 306 (2004) 666. https://doi.org/10.1126/science.1102896

4 C. C. Liu, H. Jiang, and Y. Yao: Phys. Rev. B 84 (2011) 195430. https://doi.org/10.1103/PhysRevB.84.195430 
5 S. Cahangirov, M. Topsakal, E. Aktürk, H. Sahin, and S. Ciraci: Phys. Rev. Lett. 102 (2009) 236804. https:// doi.org/10.1103/PhysRevLett.102.236804

6 Y. Xu, B. Yan, H.-J. Zhang, J. Wang, G. Xu, P. Tang, W. Duan, and S.-C. Zhang: Phys. Rev. Lett. 111 (2013) 136804. https://doi.org/10.1103/PhysRevLett.111.136804

7 B. van den Broek, M. Houssa, E. Scalise, G. Pourtois, V. V. Afanas'ev, and A. Stesmans: 2D Mater. 1 (2014) 021004. https://doi.org/10.1088/2053-1583/1/2/021004

8 Z. Ni, Q. Liu, K. Tang, J. Zheng, J. Zhou, R. Qin, Z. Gao, D. Yu, and J. Lu: Nano Lett. 12 (2012) 113. https:// doi.org/10.1021/n1203065e

9 C. N. Chen, W. L. Su, M. E. Lee, J. Y. Jen, and Y. Li: Jpn. J. Appl. Phys. 50 (2011) 060201. https://doi. org/10.1143/JJAP.50.060201

10 C. N. Chen, S. H. Chang, W. L. Su, J. Y. Jen, and Y. Li: Math. Comput. Model. 58 (2013) 282. https://doi. org/10.1016/j.mcm.2012.11.010

11 S. Datta: Superlattices Microstruct. 28 (2000) 253.

12 S. Datta: Electronic Transport in Mesoscopic Systems (Cambridge University Press, Cambridge, 1995).

13 R. Venugopal, Z. Ren, S. Datta, M. S. Lundstrom, and D. Jovanovic: J. Appl. Phys. 92 (2002) 3730. https://doi. org/10.1063/1.1503165

14 R. Golizadeh-Mojarad and S. Datta: Phys. Rev. B 75 (2007) 081301. https//doi.org/10.1103/PhysRevB.75.081301

15 S. K. Chin, K. T. Lam, D. Seah, and G. C. Liang: Nanoscale Res. Lett. 7 (2012) 114. https://doi. org/10.1186/1556-276X-7-114

16 A. Yazdanpanah, M. Pourfath, M. Fathipour, H. Kosina, and S. Selberherr: IEEE Trans. Electron Devices 59 (2012) 433. https://doi.org/10.1109/TED.2011.2173690

17 K. S. Dy, S. Y. Wu, and T. Sprathn: Phys. Rev. B 20 (1979) 4237. https://doi.org/10.1103/PhysRevB.20.4237

18 D. H. Lee and J. D. Joannopoulos: Phys. Rev. B 23 (1981) 4988. https://doi.org/10.1103/PhysRevB.23.4988

19 C. Caroli, R. Combescot, P. Nozieres, and D. Saint-James: J. Phys. C 4 (1971) 916. https://doi.org/10.1088/00223719/4/8/018

20 J. A. Stovneng and P. Lipavsky: Phys. Rev. B 49 (1994) 16494. https://doi.org/10.1103/PhysRevB.49.16494

21 G. Kim, H. Suh, and E. Lee: Phys. Rev. B 52 (1995) 2632. https://doi.org/10.1103/PhysRevB.52.2632

22 A. Umerski: Phys. Rev. B 55 (1997) 5266. https://doi.org/10.1103/PhysRevB.55.5266

23 M. Ogawa, T. Sugano, and T. Miyoshi: Solid State Electron. 44 (2000) 1939. https://doi.org/10.1016/S00381101(00)00174-X

24 D. Z.-Y. Ting: Microelectron. J. 30 (1999) 985. https://doi.org/10.1016/S0026-2692(99)00065-8

25 T. B. Boykin: Phys. Rev. B 54 (1996) 8107. https://doi.org/10.1103/PhysRevB.54.8107

26 Y. C. Chang and J. N. Schulman: Phys. Rev. B 25 (1982) 3975. https://doi.org/10.1103/PhysRevB.25.3975

27 M. Ogawa, T. Sugano, and T. Miyoshi: Physica E 7 (2000) 840. https://doi.org/10.1016/S1386-9477(00)00073-4

28 J. N. Schulman and Y. C. Chang: Phys. Rev. B 27 (1983) 2346. https://doi.org/10.1103/PhysRevB.27.2346

29 D. Z.-Y. Ting, E. T. Yu, and T. C. McGill: Phys. Rev. B 45 (1992) 3583. https://doi.org/10.1103/ PhysRevB.45.3583

30 J. C. Chiang and Y. C. Chang: J. Appl. Phys. 73 (1993) 2402. https://doi.org/10.1063/1.353094

31 C. N. Chen, W. J. Luo, F. L. Shyu, H. C. Chung, C. Y. Lin, and J. Y. Wu: Mod. Phys. Lett. B 32 (2018) 1750355. https://doi.org/10.1142/S0217984917503559

32 S. Datta: Quantum Transport: Atom to Transistor (Cambridge University Press, Cambridge, 2005).

33 V. H. Nguyen, V. N. Do, A. Bournel, V. L. Nguyen, and P. Dollfus: J. Appl. Phys. 106 (2009) 053710. https:// doi.org/10.1063/1.3212984

34 Y. T. Zhang, Q. F. Sun, and X. C. Xie : J. Appl. Phys. 109 (2011) 123718. https://doi.org/10.1063/1.3599930

35 A. Orlof, J. Ruseckas, and I. V. Zozoulenko: Phys. Rev. B 88 (2013) 125409. https://doi.org/10.1103/ PhysRevB.88.125409

36 Y. Wu and P. A. Childs: Nanoscale Res. Lett. 6 (2011) 62.

37 B. Novakovic, R. Akis, and I. Knezevic: Phys. Rev. B 84 (2011) 195419. https://doi.org/10.1103/ PhysRevB.84.195419

38 H. B. Ribeiro, K. Sato, G. S. N. Eliel, E. A. T. de Souza, C. C. Lu, P. W. Chiu, R. Saito, and M. A. Pimenta: Carbon 90 (2015) 138. https://doi.org/10.1016/j.carbon.2015.04.005

39 Y. Yang, G. Fedorov, S. E. Shafranjuk, T. M. Klapwijk, B. K. Cooper, R. M. Lewis, C. J. Lobb, and P. Barbara: Nano Lett. 15 (2015) 7859. https://doi.org/10.1021/acs.nanolett.5b02564

40 X. Lin and J. Ni : J. Appl. Phys. 117 (2015) 164305. https://doi.org/10.1063/1.4919223

41 K. Y. Li, W. J. Luo, and S. J. Wei: Appl. Sci. 10 (2020) 3991. https://doi.org/10.3390/app10113991

42 K. Y. Li, W. J. Luo, X. H. Hong, S. J. Wei, and P. H. Tai: IEEE Access 8 (2020) 28988. https://doi.org /10.1109/ ACCESS.2020.2972580

43 K. Y. Li, W. J. Luo, J. Z. Huang, Y. C. Chan, Pratikt, and D. Faridah: Appl. Sci. 7 (2017) 420. https://doi. org/10.3390/app7040420 


\section{About the Authors}

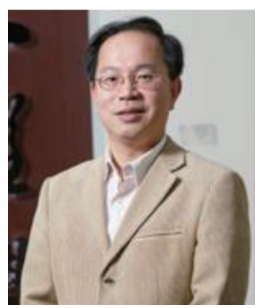

Win-Jet Luo earned his master's degree in engineering in 1994 and his Ph.D. degree in engineering in 2000 from National Chen Kung University (NCKU), Taiwan. At present, he is a professor in the Graduate Institute of Precision Manufacturing, National Chin Yi University of Technology (NCUT). His research has mainly focused on computational fluid dynamics, micro-electromechanical systems, ventilation, energy saving, fuel cells, and microsensors. He has published more than 70 research papers in prestigious international journals, and he has been invited to serve as a reviewer for journals.(wjluo@ncut.edu.tw)

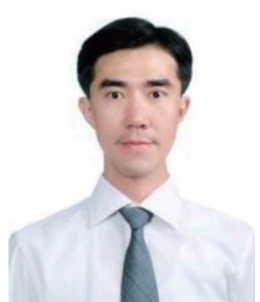

Wei-Ta Chien earned his master's degree in materials science and engineering in 1999 from National Taiwan University of Science and Technology and his $\mathrm{PhD}$ degree in microelectronics in 2011 from National Cheng-Kung University, Taiwan. At present, Chien is an assistant professor in the Department of Refrigeration, Air-Conditioning and Engineering at Far East University.

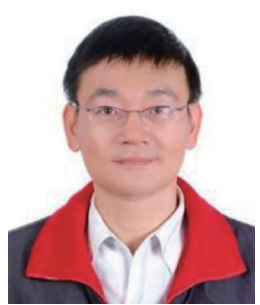

Hau-Chen Yen was born in Kaohsiung, Taiwan, R.O.C., in 1964. He received his M.S. degree in computer and information engineering from Tamkang University, Taipei, Taiwan, R.O.C., and Ph.D. degree from National Sun Yat-sen University, Kaohsiung, Taiwan, R.O.C., in 1992 and 2003, respectively. His research interests include power electronic converters and their applications.

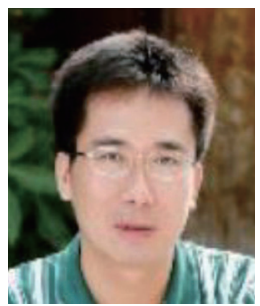

Chun-Nan Chen was born in Taitung, Taiwan, R.O.C., in 1963. He received his M.S. degree in electrical and computer engineering from Arizona State University, United States, and Ph.D. degree from NCKU, Taiwan., R.O.C., in 1989 and 2002, respectively. His research interests include quantum physics and their applications.

\section{Appendix A: Hamiltonian of Monolayer Group-IVA Elements}

For a monolayer group-IVA element structure, the Hamiltonian with the $\alpha_{\beta}^{\tau}$ orbital, which is calculated using the tight-binding model with the SOC effect and only nearest-neighbor interactions, can be written as

$$
H_{\text {monolayer }}(\boldsymbol{k})=H_{t b}(\boldsymbol{k})+H_{s o c}
$$

where $H_{t b}$ is the tight-binding Hamiltonian and $H_{s o c}$ is the SOC Hamiltonian. 
The tight-binding Hamiltonian $H_{t b}$ with orbital ordering $\left\{\alpha_{A}^{\uparrow}, \alpha_{B}^{\uparrow}, \alpha_{A}^{\downarrow}, \alpha_{B}^{\downarrow}\right\}$ and $\alpha(=\{z, y, x, s\})$ can be expressed in the $16 \times 16$ matrix form as

$$
H_{t b}(\boldsymbol{k})=\left[\begin{array}{cc}
H_{t b}^{\uparrow \uparrow} & 0 \\
0 & H_{t b}^{\downarrow \downarrow}
\end{array}\right],
$$

with

$$
H_{t b}^{\uparrow \uparrow(o r \downarrow)}=\left[\begin{array}{cccccccc}
0 & 0 & 0 & 0 & H_{z z}^{A} & H_{z y}^{A} & H_{z x}^{A} & H_{z s}^{A} \\
0 & 0 & 0 & 0 & H_{y z}^{A} & H_{y y}^{A} & H_{y x}^{A} & H_{y s}^{A} \\
0 & 0 & 0 & 0 & H_{x z}^{A} & H_{x y}^{A} & H_{x x}^{A} & H_{x s}^{A} \\
0 & 0 & 0 & \Delta & H_{s z}^{A} & H_{s y}^{A} & H_{s x}^{A} & H_{s s}^{A} \\
H_{z z}^{B} & H_{z y}^{B} & H_{z x}^{B} & H_{z s}^{B} & 0 & 0 & 0 & 0 \\
H_{y z}^{B} & H_{y y}^{B} & H_{y x}^{B} & H_{y s}^{B} & 0 & 0 & 0 & 0 \\
H_{x z}^{B} & H_{x y}^{B} & H_{x x}^{B} & H_{x s}^{B} & 0 & 0 & 0 & 0 \\
H_{s z}^{B} & H_{s y}^{B} & H_{s x}^{B} & H_{s s}^{B} & 0 & 0 & 0 & \Delta
\end{array}\right],
$$

where

$$
\begin{aligned}
& H_{z z}^{A}=\left(H_{z z}^{B}\right)^{*}=\left(V_{p p \sigma}-V_{p p \pi}\right) \cos ^{2} \theta T_{3}+V_{p p \pi} T_{3}, \\
& H_{y y}^{A}=\left(H_{y y}^{B}\right)^{*}=\left(V_{p p \sigma}-V_{p p \pi}\right) \sin ^{2} \theta T_{5}+V_{p p \pi} T_{3}, \\
& H_{x x}^{A}=\left(H_{x x}^{B}\right)^{*}=\frac{3}{4}\left(V_{p p \sigma}-V_{p p \pi}\right) \sin ^{2} \theta T_{1}+V_{p p \pi} T_{3}, \\
& H_{y z}^{A}=\left(H_{y z}^{B}\right)^{*}=\left(V_{p p \sigma}-V_{p p \pi}\right) \sin \theta \cos \theta T_{4}, \\
& H_{x z}^{A}=\left(H_{x z}^{B}\right)^{*}=\frac{\sqrt{3}}{2}\left(V_{p p \sigma}-V_{p p \pi}\right) \sin \theta \cos \theta T_{2}, \\
& H_{x y}^{A}=\left(H_{x y}^{B}\right)^{*}=\frac{\sqrt{3}}{4}\left(V_{p p \sigma}-V_{p p \pi}\right) \sin ^{2} \theta T_{2}, \\
& H_{s z}^{A}=\left(-H_{s z}^{B}\right)^{*}=V_{s p \sigma} \cos \theta T_{3}, \\
& H_{s y}^{A}=\left(-H_{s y}^{B}\right)^{*}=V_{s p \sigma} \sin \theta T_{4}, \\
& H_{s x}^{A}=\left(-H_{s x}^{B}\right)^{*}=\frac{\sqrt{3}}{2} V_{s p \sigma} \sin \theta T_{2}, \\
& H_{s s}^{A}=\left(H_{s s}^{B}\right)^{*}=V_{s s \sigma} T_{3},
\end{aligned}
$$




$$
\begin{aligned}
& T_{1}=2 e^{i k_{\|} b / 2} \cos \frac{1}{2} k_{\perp} a, \\
& T_{2}=2 i e^{i k_{\|} b / 2} \sin \frac{1}{2} k_{\perp} a, \\
& T_{3}=2 e^{i k_{\|} b / 2} \cos \frac{1}{2} k_{\perp} a+e^{-i k_{\|} b}, \\
& T_{4}=e^{i k_{\|} b / 2} \cos \frac{1}{2} k_{\perp} a-e^{-i k_{\|} b}, \\
& T_{5}=\frac{1}{2} e^{i k_{\|} b / 2} \cos \frac{1}{2} k_{\perp} a+e^{-i k_{\|} b},
\end{aligned}
$$

$\boldsymbol{k}$ is a wave vector $\left(k_{\perp}, k_{\|}\right), \theta$ is the angle between the bond and the $z$ direction, $a(=\sqrt{3} b)$ is the lattice constant, $b$ is the bond length projected on the layer plane, and a total of five interaction parameters exist, namely, $\Delta, V_{p p \sigma}, V_{p p \pi}, V_{s p \sigma}$, and $V_{s s \sigma} . \Delta$ is related to the on-site energy difference between the $s$ and $p$ orbitals, while the remaining four parameters represent the nearest-neighbor interactions.

The SOC Hamiltonian $H_{S O C}$ with orbital ordering $\left\{\alpha_{A}^{\uparrow}, \alpha_{B}^{\uparrow}, \alpha_{A}^{\downarrow}, \alpha_{B}^{\downarrow}\right\}$ and $\alpha(=\{z, y, x, s\})$ can be expressed in the $16 \times 16$ matrix form as

$$
H_{s o c}=\left[\begin{array}{cc}
h_{s o c}^{\uparrow \uparrow} & h_{s o c}^{\uparrow \downarrow} \\
h_{\text {soc }}^{\downarrow \uparrow} & h_{\text {soc }}^{\downarrow \downarrow}
\end{array}\right],
$$

with

$$
h_{s o c}=\left[\begin{array}{cccccccc}
0 & i \sigma_{x} & -i \sigma_{y} & 0 & 0 & 0 & 0 & 0 \\
-i \sigma_{x} & 0 & i \sigma_{z} & 0 & 0 & 0 & 0 & 0 \\
i \sigma_{y} & -i \sigma_{z} & 0 & 0 & 0 & 0 & 0 & 0 \\
0 & 0 & 0 & 0 & 0 & 0 & 0 & 0 \\
0 & 0 & 0 & 0 & 0 & i \sigma_{x} & -i \sigma_{y} & 0 \\
0 & 0 & 0 & 0 & -i \sigma_{x} & 0 & i \sigma_{z} & 0 \\
0 & 0 & 0 & 0 & i \sigma_{y} & -i \sigma_{z} & 0 & 0 \\
0 & 0 & 0 & 0 & 0 & 0 & 0 & 0
\end{array}\right] \times \frac{\xi_{0}}{2},
$$

where $\sigma_{x}, \sigma_{y}$, and $\sigma_{z}$ are the Pauli spin matrices and $\xi_{0}$ is the strength of the SOC effect. The parameters in this appendix are shown in Tables I and II of Ref. 4.

\section{Appendix B: Hamiltonian of Zigzag-edge Group-IVA Nanoribbon}

The z4ANR Hamiltonian, which is calculated using the tight-binding model with the SOC effect and only nearest-neighbor interactions, in the $\mid k_{\perp}, j, \alpha_{\beta}^{\tau}>$ basis can be written in the $N \times N$ block matrix form as $(j=1,2, \ldots, N)$ 


$$
H_{z 4 A N R}\left(k_{\perp}\right)=\left[\begin{array}{ccccc}
h_{1} & h_{2}^{+} & 0 & \cdots & 0 \\
h_{2} & h_{1} & h_{2}^{+} & 0 & \vdots \\
0 & h_{2} & h_{1} & \ddots & 0 \\
\vdots & 0 & \ddots & \ddots & h_{2}^{+} \\
0 & \cdots & 0 & h_{2} & h_{1}
\end{array}\right],
$$

where $h_{1}$ and $h_{2}$ are $16 \times 16$ matrices with orbital ordering $\left\{\alpha_{A}^{\uparrow}, \alpha_{B}^{\uparrow}, \alpha_{A}^{\downarrow}, \alpha_{B}^{\downarrow}\right\}$ and $\alpha(=\{z, y, x, s\})$.

The $h_{1}$ matrix can be written in the $16 \times 16$ matrix form as

$$
h_{1}=h_{t b}\left(k_{\perp}\right)+H_{s o c},
$$

where $H_{S O C}$ is given by Eq. (A4), $h_{t b}$ has basically the same form as $H_{t b}$ in Eq. (A2), but $T_{i=1-5)}$ must be changed to $T_{1}=T_{3}=2 T_{4}=4 T_{5}=2 \cos \frac{1}{2} k_{\perp} a$ and $T_{2}=2 i \sin \frac{1}{2} k_{\perp} a$.

The $h_{2}$ matrix can be written in the $16 \times 16$ matrix form as

$$
h_{2}=\left[\begin{array}{cc}
h_{2}^{\uparrow \uparrow} & 0 \\
0 & h_{2}^{\downarrow \downarrow}
\end{array}\right],
$$

with

$$
h_{2}^{\uparrow \uparrow(o r \downarrow \downarrow)}=\left[\begin{array}{cccccccc}
0 & 0 & 0 & 0 & h_{z z}^{A} & h_{z y}^{A} & 0 & h_{z s}^{A} \\
0 & 0 & 0 & 0 & h_{y z}^{A} & h_{y y}^{A} & 0 & h_{y s}^{A} \\
0 & 0 & 0 & 0 & 0 & 0 & h_{x x}^{A} & 0 \\
0 & 0 & 0 & 0 & h_{s z}^{A} & h_{s y}^{A} & 0 & h_{s s}^{A} \\
0 & 0 & 0 & 0 & 0 & 0 & 0 & 0 \\
0 & 0 & 0 & 0 & 0 & 0 & 0 & 0 \\
0 & 0 & 0 & 0 & 0 & 0 & 0 & 0 \\
0 & 0 & 0 & 0 & 0 & 0 & 0 & 0
\end{array}\right],
$$

where

$$
\begin{aligned}
& h_{z z}^{A}=\left(V_{p p \sigma}-V_{p p \pi}\right) \cos ^{2} \theta+V_{p p \pi}, \\
& h_{y y}^{A}=\left(V_{p p \sigma}-V_{p p \pi}\right) \sin ^{2} \theta+V_{p p \pi}, \\
& h_{x x}^{A}=V_{p p \pi}, \\
& h_{y z}^{A}=h_{z y}^{A}=-\left(V_{p p \sigma}-V_{p p \pi}\right) \sin \theta \cos \theta, \\
& h_{s z}^{A}=-h_{z s}^{A}=V_{s p \sigma} \cos \theta,
\end{aligned}
$$




$$
\begin{aligned}
& h_{s y}^{A}=-h_{y s}^{A}=-V_{s p \sigma} \sin \theta, \\
& h_{s s}^{A}=V_{s s \sigma} .
\end{aligned}
$$

\section{Appendix C: Hamiltonians of Zigzag-edge Group-IVA Nanoribbon Between Adjacent Layers}

The Hamiltonians $H_{\sigma, \sigma}$ and $H_{\sigma, \sigma \pm 1}$ of a z4ANR with $N$ zigzag lines, which are calculated using the tight-binding model with the SOC effect and only nearest-neighbor interactions, in the $\mid \sigma, j, \alpha_{\beta}^{\tau}>$ layer basis can be written in the $N \times N$ block matrix forms $(j=1,2, \ldots, N)$

$$
H_{\sigma, \sigma}=\left[\begin{array}{ccccc}
g_{1} & g_{2}^{+} & 0 & \cdots & 0 \\
g_{2} & g_{1} & g_{2}^{+} & 0 & \vdots \\
0 & g_{2} & g_{1} & \ddots & 0 \\
\vdots & 0 & \ddots & \ddots & g_{2}^{+} \\
0 & \cdots & 0 & g_{2} & g_{1}
\end{array}\right]
$$

and

$$
H_{\sigma, \sigma \pm 1}=\left[\begin{array}{ccccc}
z_{ \pm} & 0 & 0 & \cdots & 0 \\
0 & z_{ \pm} & 0 & 0 & \vdots \\
0 & 0 & z_{ \pm} & \ddots & 0 \\
\vdots & 0 & \ddots & \ddots & 0 \\
0 & \cdots & 0 & 0 & z_{ \pm}
\end{array}\right],
$$

where each of the block matrix elements is $16 \times 16$-dimensional, in the orbital ordering $\left\{\alpha_{A}^{\uparrow}, \alpha_{B}^{\uparrow}, \alpha_{A}^{\downarrow}, \alpha_{B}^{\downarrow}\right\}$ and $\alpha(=\{z, y, x, s\})$.

The $g_{1}$ matrix can be written in the $16 \times 16$ matrix form as

$$
g_{1}=H_{s o c}+\left[\begin{array}{cc}
g^{\uparrow \uparrow}-E & 0 \\
0 & g^{\downarrow \downarrow}-E
\end{array}\right],
$$

where $E$ is the injected electron energy, $H_{S O C}$ is given by Eq. (A4), and

$$
g^{\uparrow \uparrow(o r \downarrow \downarrow)}=\left[\begin{array}{cccccccc}
0 & 0 & 0 & 0 & 0 & 0 & 0 & 0 \\
0 & 0 & 0 & 0 & 0 & 0 & 0 & 0 \\
0 & 0 & 0 & 0 & 0 & 0 & 0 & 0 \\
0 & 0 & 0 & \Delta & 0 & 0 & 0 & 0 \\
0 & 0 & 0 & 0 & 0 & 0 & 0 & 0 \\
0 & 0 & 0 & 0 & 0 & 0 & 0 & 0 \\
0 & 0 & 0 & 0 & 0 & 0 & 0 & 0 \\
0 & 0 & 0 & 0 & 0 & 0 & 0 & \Delta
\end{array}\right] .
$$


The $g_{2}$ matrix can be expressed in the $16 \times 16$ matrix form, which has the same form as the $h_{2}$ matrix, i.e., $g_{2}=h_{2}$.

The $z_{ \pm}$matrix can be written in the $16 \times 16$ matrix form as

$$
z_{ \pm}=\left[\begin{array}{cc}
z_{ \pm}^{\uparrow \uparrow} & 0 \\
0 & z_{ \pm}^{\downarrow \downarrow}
\end{array}\right],
$$

with

$$
z_{ \pm}^{\uparrow \uparrow(o r \downarrow \downarrow)}=\left[\begin{array}{cccccccc}
0 & 0 & 0 & 0 & z_{z z}^{A} & z_{z y}^{A} & z_{z x}^{A} & z_{z s}^{A} \\
0 & 0 & 0 & 0 & z_{y z}^{A} & z_{y y}^{A} & z_{y x}^{A} & z_{y s}^{A} \\
0 & 0 & 0 & 0 & z_{x z}^{A} & z_{x y}^{A} & z_{x x}^{A} & z_{x s}^{A} \\
0 & 0 & 0 & 0 & z_{s z}^{A} & z_{s y}^{A} & z_{s x}^{A} & z_{s s}^{A} \\
z_{z z}^{B} & z_{z y}^{B} & z_{z x}^{B} & z_{z s}^{B} & 0 & 0 & 0 & 0 \\
z_{y z}^{B} & z_{y y}^{B} & z_{y x}^{B} & z_{y s}^{B} & 0 & 0 & 0 & 0 \\
z_{x z}^{B} & z_{x y}^{B} & z_{x x}^{B} & z_{x s}^{B} & 0 & 0 & 0 & 0 \\
z_{s z}^{B} & z_{s y}^{B} & z_{s x}^{B} & z_{s s}^{B} & 0 & 0 & 0 & 0
\end{array}\right],
$$

where

$$
\begin{aligned}
& z_{z z}^{A}=z_{z z}^{B}=\left(V_{p p \sigma}-V_{p p \pi}\right) \cos ^{2} \theta+V_{p p \pi}, \\
& z_{y y}^{A}=z_{y y}^{B}=\frac{1}{4}\left(V_{p p \sigma}-V_{p p \pi}\right) \sin ^{2} \theta+V_{p p \pi}, \\
& z_{x x}^{A}=z_{x x}^{B}=\frac{3}{4}\left(V_{p p \sigma}-V_{p p \pi}\right) \sin ^{2} \theta+V_{p p \pi}, \\
& z_{y z}^{A}=z_{z y}^{A}=z_{z y}^{B}=z_{y z}^{B}=\frac{1}{2}\left(V_{p p \sigma}-V_{p p \pi}\right) \sin \theta \cos \theta, \\
& z_{x z}^{A}=z_{z x}^{A}=-z_{z x}^{B}=-z_{x z}^{B}= \pm \frac{\sqrt{3}}{2}\left(V_{p p \sigma}-V_{p p \pi}\right) \sin \theta \cos \theta, \\
& z_{x y}^{A}=z_{y x}^{A}=-z_{y x}^{B}=-z_{x y}^{B}= \pm \frac{\sqrt{3}}{4}\left(V_{p p \sigma}-V_{p p \pi}\right) \sin ^{2} \theta, \\
& z_{s z}^{A}=-z_{z s}^{A}=z_{z s}^{B}=-z_{s z}^{B}=V_{s p \sigma} \cos \theta, \\
& z_{s y}^{A}=-z_{y s}^{A}=z_{y s}^{B}=-z_{s y}^{B}=\frac{1}{2} V_{s p \sigma} \sin \theta, \\
& z_{s x}^{A}=-z_{x s}^{A}=-z_{x s}^{B}=z_{s x}^{B}= \pm \frac{\sqrt{3}}{2} V_{s p \sigma} \sin \theta, \\
& z_{s s}^{A}=z_{s s}^{B}=V_{s s \sigma} .
\end{aligned}
$$

